W. Thiel ${ }^{1}$

L. Kowalzick ${ }^{2}$

\section{Maximalausprägung einer Psoriasis vulgaris cum pustulatione}

Signifikante Besserung des Hautbildes durch Efalizumab (Raptiva ${ }^{\circledR}$ )

Severe Plaque Psoriasis cum pustulatione - Significant Improvement by Treatment with Efalizumab (Raptiva ${ }^{\circledR}$ )

Zusammenfassung

Kürzlich wurde über das gute therapeutische Ansprechen von Patienten mit generalisierter pustulöser Psoriasis auf Infliximab berichtet. Wir berichten über eine 55 -jährige Patientin mit langjährig bestehender, häufig schwer rezidivierender Psoriasis vulgaris cum pustulatione, die nach Versagen oder Unverträglichkeit anderer systemischer Psoriasis-Therapien nach einer initialen Infliximab-Infusion mittels regelmäßiger s.c. Efalizumab-Injektionen über einen längeren Zeitraum stabilisiert werden konnte.
Abstract

Recently, the good clinical therapeutic effect of infliximab in patients with pustular psoriasis was demonstrated. We report on a 55 years old female patient with longstanding frequently relapsing plaque psoriasis cum pustulatione. Because of failure or intolerance of other systemic antipsoriatic therapies we initiated after one single infusion of infliximab a treatment with regular subcutaneous injections of efalizumab which resulted in persisting remission of the psoriasis.

\section{Einleitung}

Untersuchungen haben gezeigt, dass besonders bei schweren Formen der Psoriasis die große Belastung für die Erkrankten vergleichbar mit der anderer schwerer Erkrankungen wie Diabetes, Rheuma, Depressionen oder Krebs ist [11]. Wie stark die Lebensqualität eingeschränkt sein kann, belegt die vorliegende Kasuistik. Bei der Patientin mit der Maximalausprägung einer Psoriasis vulgaris cum pustulatione reichte eine topische Behandlung nicht aus und bisher verfügbare systemische Psoriasis-Therapien erwiesen sich in ihrer Anwendung ineffektiv bzw. durch toxische Effekte limitiert. Kürzlich wurde über das gute therapeutische Ansprechen von Patienten mit pustulöser Psoriasis auf Infliximab berichtet [5,9]. Auch im vorliegenden Fall konnte erst die Behandlung mit Biologics wie Efalizumab (Raptiva ${ }^{\circledR}$ ) die Situation der Patientin wesentlich verbessern und führte innerhalb kurzer Zeit zur fast völligen Symptomfreiheit.

\section{Kasuistik}

\section{Anamnese und Befund}

Erstmals traten bei der 55-jährigen Patientin vor 11 Jahren schwerste Hauterscheinungen auf. Zudem kam es zu Schmerzen in den Kniegelenken. Die Psoriasis-Herde verschlimmerten sich in der Folgezeit unter Bildung schmerzhafter Pusteln. Auch auf Drängen der Angehörigen erfolgten wiederholt - ca. zwei- bis viermal jährlich - stationäre Aufnahmen, da die Patientin aufgrund panzerartiger Schuppenkrusten mit darunter liegenden Pusteln und Erosionen mit zum Teil schmerzhaften Rhagaden an den Füßen zunehmend in ihrer Beweglichkeit eingeschränkt war [12]. Nicht nur die Beweglichkeit erwies sich als reduziert, sondern das gesamte Allgemeinbefinden der Patientin war extrem reduziert. Neben den Hauterscheinungen lagen Nageldystrophien zum Teil mit subungualen Pusteln bis zur Onycholyse vor (Abb. 1). Verursacht oder begünstigt wurde diese Maximal- 


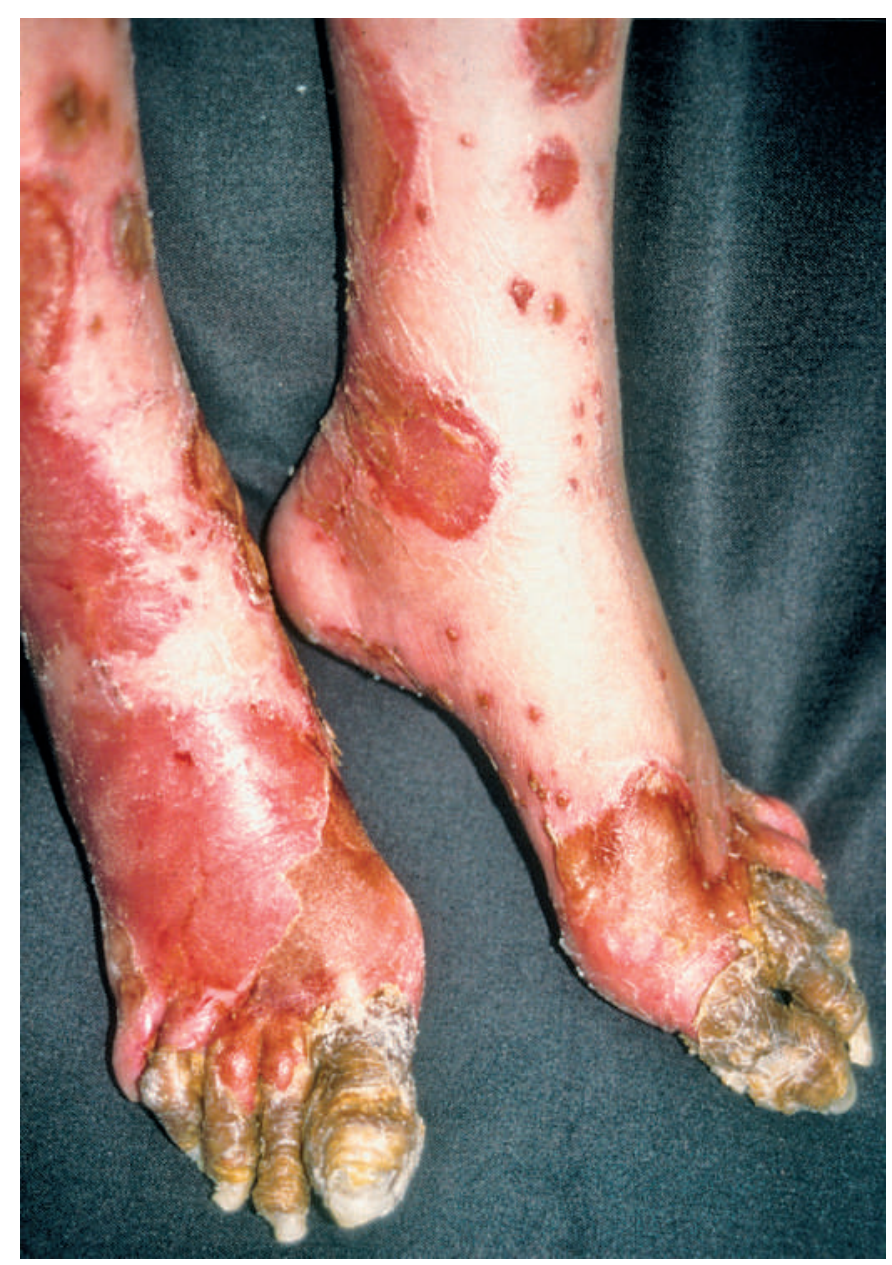

Abb. 1 Das Hautbild wies insbesondere im Bereich der unteren Extremitäten und Füße schwersten psoriatrischen Befall mit Pusteln auf.

ausprägung einer Psoriasis vulgaris cum pustulationem durch fokale Infekte, hormonelle Störungen, Alkohol- und Nikotinabusus auf dem Boden einer unbehandelten Psoriasis. Seit mittlerweile 6 Jahren wurde regelmäßig versucht, das dramatische Krankheitsgeschehen durch systemische Therapien positiv zu beeinflussen. Allerdings konnte durch eine Retinoid-Therapie nur ein mäßiger Effekt erreicht werden. Nachdem die Dosis des Medikaments reduziert wurde, kam es erneut zu einer Exazerbation. Ein Therapieversuch mit Fumarsäureester rund ein Jahr später erwies sich als ebenso ungeeignet: Obwohl der Therapieeffekt nur mäßig ausgeprägt war, kam es zu schweren Nebenwirkungen. In erster Linie handelte es sich dabei um gastrointestinale Beschwerden sowie Fieber und Schüttelfrost. Eine darauf folgende Ciclosporin A-Therapie konnte zwar die Symptome gut eindämmen, erwies sich aufgrund der Nierenwerte als nicht weiter durchführbar. Daher wurde auf eine Methotrexat-Behandlung ausgewichen. Unter dieser Therapie trat ein progredienter Verlauf auf.

\section{Therapie und Verlauf}

In Anbetracht der ausgeschöpften und erfolglosen Möglichkeiten der systemischen Therapien wurde beim nächsten stationären Aufenthalt mit einer Infliximab (Remicade ${ }^{\circledR}$ )-Behandlung begonnen. Die intravenöse Infusion von $200 \mathrm{mg}$ (entsprechend etwa $5 \mathrm{mg} / \mathrm{kg}$ Körpergewicht) des TNF-alpha-Antikörpers bewirkte eine deutliche und rasche Besserung des Hautbildes. Allerdings

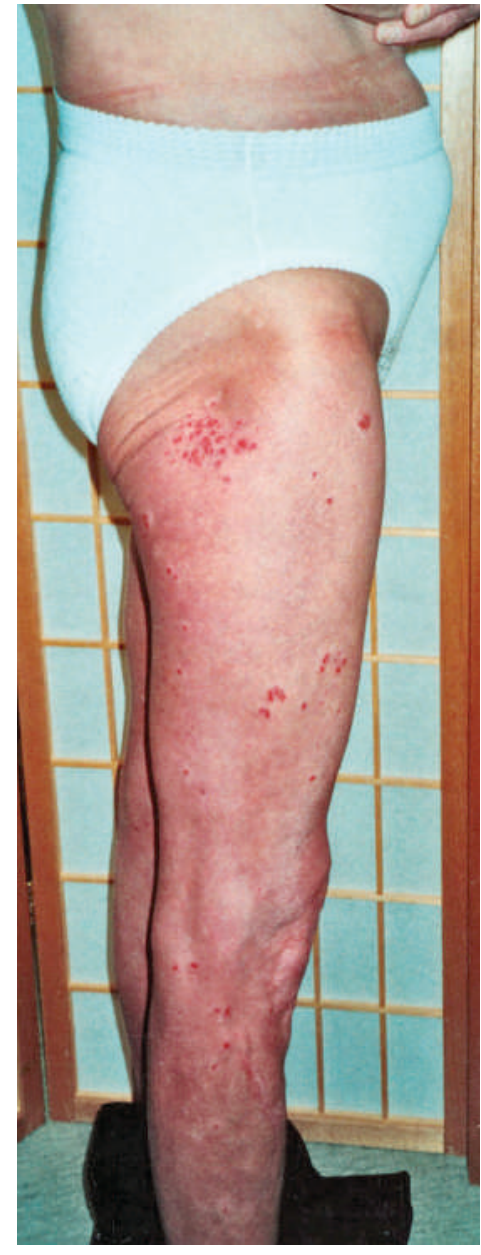

Abb. 2 Starke Besserung des Hautbildes nach Behandlung mit Efalizumab. traten im Verlauf des stationären Aufenthaltes im Bereich der Beine wieder kleine erythematosquamöse Herde an den Oberund Unterschenkeln auf. Damit eine nachhaltig wirksame Theiapie auch unter ambulanten Bedingungen weitergeführt werden konnte, erfolgte anschließend nach 2 Wochen eine Fortsetzung der Therapie mit Efalizumab (Raptiva ${ }^{\circledR}$ ) mit wöchentlichen Injektionen von initial $30 \mathrm{mg}$, später $40 \mathrm{mg}$. Dieser selektiv und reversibel wirkende T-Zell-Modulator erlaubt die subkutane Verabreichung und ermöglicht damit eine gute ambulante Therapie. Nach drei Monaten Behandlung unter Efalizumab ließ sich ein sehr guter Behandlungserfolg verzeichnen, nur an beiden Oberschenkeln kamen lateral vereinzelte punkt- bzw. fleckförmige erythematosquamöse Herde vor (Abb. 2). Das Hautbild war ansonsten nahezu erscheinungsfrei. Die Pusteln sind ganz verschwunden. Die Gelenkbeschwerden hatten ebenfalls vollständig abgenommen.

\section{Diskussion}

Am Beispiel dieser Patientin wird deutlich, dass die neuen Biologics selbst bei Betroffenen mit schwerer Psoriasis und fehlender Compliance nicht nur eine Besserung des Hautbildes sondern auch der Lebensqualität erbringen können. Besonders erweist sich Efalizumab von Vorteil: Dieser speziell für die Psoriasis entwickelte Wirkstoff, ein humanisierter monoklonaler Antikörper 
gegen CD 11a, einer Untereinheit des Leukozyten-funktionsassoziierten Antigens 1 (LFA-1), kann zielgerichtet die T-Zell-Aktivität modulieren, indem er die Adhäsion von T-Zellen an Endothelzellen in den Blutgefäßen der Haut blockiert. Zudem hemmt Efalizumab auch die sekundäre Aktivierung der T-Zellen in der Haut [1]. Auf diese Weise werden die inflammatorischen Prozesse gestoppt und es kommt zu einer Normalisierung der gestörten Differenzierung und Proliferation der Keratinozyten. Aufgrund dieses Wirkmechanismus zeichnet sich Efalizumab durch ein sehr gutes Wirksamkeits- und Sicherheitsprofil aus. Der Wirkstoff wird einmal wöchentlich subkutan verabreicht, dadurch erübrigen sich häufige Arztbesuche und komplizierte Behandlungsschemata. Bei moderater bis schwerer Plaquepsoriasis erreichten 22-28\% der behandelten Patienten nach 3-monatiger Behandlung eine Reduktion ihres PASI-Ausgangswertes von mindestens $75 \%$ [3,6]. Die Therapie bedeutet für die meisten Patienten einen oft dramatischen Gewinn an Lebensqualität, weil viele von ihnen erstmals seit langer Zeit beinahe oder vollständig frei von Hauterscheinungen sind [10]. Bei Patienten, die in den ersten drei Monaten auf Efalizumab ansprachen, erreichten bei Fortsetzung der Therapie nach 6 Monaten $44-53 \%[7,8]$ und nach 26 Monaten 56\% [4] der Patienten eine PASI-Abnahme von mindestens 75\% des Ausgangswertes. Nach Absetzen einer Biologics-Therapie wurden aber in Einzelfällen auch nach Efalizumab pustulöse Psoriasis-Rezidive beobachtet [2].

\section{Literatur}

${ }^{1}$ Dedrick RL, Walicke P, Garovoy M. Anti-adhesion antibodies efalizumab, a human anti-CD11a monoclonal antibody. Transpl Immunol 2002; 9: $181-186$

${ }^{2}$ Gaylor ML, Duvic M. Generalized pustular psoriasis following withdrawal of efalizumab. J Drugs Dermatol 2004; 3: 77-79

${ }^{3}$ Gordon KB, Papp KA, Hamilton TK, Walicke PA, Dummer W, Li N, Bersanahan BW, Menter A. Efalizumab for Patients with moderate to severe plaque psoriasis: a randomized controlled trial. JAMA 2004; 291: $3133-3135$

${ }^{4}$ Gottlieb AB, Hamilton T, Caro I, Kwon P, Compton PG, Leonardi CL. Long-term continous efalizumab therapy in patients with moderate to severe plaque psoriasis: updated results from an ongoing trial. J Am Acad Dermatol 2006; 54: S154-S163

${ }^{5}$ Kowalzick L, Mischke D, Blum R, Iliev D. Erfolgreiche Therapie einer Psoriasis pustulosa generalisata von Zumbusch mit Anti-Tumornekrosefaktor-Alpha-Antikörper (Infliximab). Aktuelle Dermatologie 2003; 29: $289-292$

${ }^{6}$ Lebwohl M, Tyring SK, Hamilton TK, Toth D, Glazer S, Tawfik NH, Walicke P, Dummer W, Wang X, Garovoy MR, Pariser D. A novel targeted T-cell modulator, efalizumab, for plaque psoriasis. N Engl J Med 2003; 349: 2004-2213

${ }^{7}$ Leonardi CL, Papp KA, Gordon KB, Menter A, Feldman SR, Caro I, Walicke PA, Compton PG, Gottlieb AB. Extended efalizumab therapy improves chronic plaque psoriasis: results from a randomized phase III trial. J Am Acad Dermatol 2005; 52: 425-433

8 Menter A, Gordon A, Carey W, Hamilton T, Glazer S, Caro I, Li N, Gulliver W. Efficacy and safety observed during 24 weeks of efalizumab therapy in patients with moderate to severe plaque psoriasis. Arch Dermatol 2005; 141: $31-38$

${ }^{9}$ Newland MR, Weinstein A, Kerdel F. Rapid response to infliximab in severe pustular psoriasis, von Zumbusch type. Int J Dermatol 2002; 41: $449-452$

${ }^{10}$ Ortonne JP, Shear N, Shumack S, Henniger E. Impact of efalizumab on patient-reported outcomes in high-need psoriasis patients: results of the international,randomized, placebo-controlled phase III clinical experience acquired with Raptiva (CLAR) trial. BMC Dermatol 2005; 16 : 13

${ }^{11}$ Rapp SR, Feldmann SR, Exum ML, Fleischer AB, Reboussin DM. Psoriasis causes as much disability as other major medical diseases. J Am Acad Dermatol 1999; 41: 401 - 407

${ }^{12}$ Suckow M, Schlehaider UK, Kowalzick L. Außergewöhnliche Maximalausprägung einer Psoriasis vulgaris cum pustulatione. Derm - Praktische Dermatologie 1997; 3: 392 - 396 